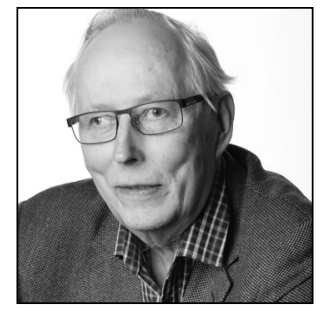

Raimo Lahti

Professor emeritus

Universität Helsinki

\title{
Die strafrechtliche Verantwortung der juristischen Person:

\author{
Rechtsvergleichende Überlegungen \\ zwischen Finnland und Estland
}

\section{Einleitung}

Der Jubilar Jaan Sootak hat das Thema über die strafrechtliche Verantwortung der juristischen Person vielmals in seiner wissenschaftlichen Produktion behandelt. ${ }^{*}{ }^{*}$ Weil diese Form der strafrechtlichen Verantwortung sowohl in Finnland als auch in Estland relativ neu ist, ist es nützlich rechtsvergleichende Gesichtspunkte darüber darzulegen, in welchen Hinsichten die Gesetzgebungslösungen und Gerichtspraxis in diesen zwei Ländern Ähnlichkeiten und Verschiedenheiten aufweisen. Ich werde in der vergleichenden Darstellung meine neuen Artikel betreffend die einschlägige finnische Regelung benutzen. ${ }^{{ }_{2}}$

Der Aufsatz von Priit Pikamäe und Jaan Sootak „Die schuldhafte strafrechtliche Verantwortung der juristischen Person“ wird als ein Referenztext in solcher Weise gebraucht, dass die wichtigsten prinzipiellen und praktischen Fragen der Thematik in entsprechender Weise wie in ihrem Aufsatz aus komparativem Gesichtswinkel erörtert werden. Die Hinweise auf die Strafgesetze Finnlands und Estlands und die Zitierungen der Gesetze stützen sich auf die Übersetzungen dieser Strafgesetze. ${ }^{* 3}$

1 Siehe insbesondere J. Sootak, E. Elkind, The concept of corporate criminal responsibility and its further developments in the Estonian case law. - Rikosoikeudellisia kirjoituksia VIII Raimo Lahdelle 12.1.2006 omistettu. Helsinki: Suomalainen Lakimiesyhdistys 2006, S. 421-435; P. Pikamäe, J. Sootak, Die schuldhafte strafrechtliche Verantwortung der juristischen Person. Theoretische Grundlagen und estnisches Gerichtspraxis. - Juridica International 2012 (XIX), S. 154-160. - DOI: https://doi.org/10.12697/issn1406-1082.

2 R. Lahti, Über die strafrechtliche Verantwortung der juristischen Person und die Organ- und Vertreterhaftung in Finnland. Rechtsstaatliches Strafen. Festschrift für Keiichi Yamanaka. Berlin: Duncker \& Humblot, Berlin 2017, 131-152. - DOI: https://doi.org/10.3790/978-3-428-54629-9 (auch im Aufsatzsammlung R. Lahti, Zur Kriminal- und Strafrechtspolitik des 21. Jahrhunderts. Berlin: de Gruyter 2019, S. 223-248. - DOI: https://doi.org/10.1515/9783110647990); R. Lahti, Finnish Report on Individual Liability for Business Involvement in International Crimes. Revue Internationale de Droit Pénal 2017 (88:1), p. 257-266.

Betreffend die finnische Regelung siehe auch D. Frände, Strafbarkeit juristischer Personen aus finnischer Sicht. H.-J. Hirsch (Hrsg.), Krise des Strafrechts und der Kriminalwissenchaften? Berlin: Duncker \& Humblot 2001, S. 228-233; M. Tolvanen, Trust, Business Ethics and Crime Prevention - Corporate Criminal Liability in Finland, - Fudan Law Journal 2009/4, S. 99-112; A. Alvesalo-Kuusi, L. Lähteenmäki, Legislating for corporate criminal liability in Finland: 22-year long debate revisited. - Journal of Scandinavian Studies in Criminology and Crime Prevention 2016 (17:1), S. 53-69. - DOI: https://doi.org/10.1080/14043858.2015.1120059.

3 Die englischsprachigen Übersetzungen sind zugänglich im Internet: betreffend das finnische Strafgesetz (fStGB; mit Änderungen bis 2015) siehe: www.finlex.fi/laki/kaannokset/1889/en18890039_20150766.pdf und betreffend das estnische 


\section{2. Über die Entstehungsgeschichte}

In Finnland wurde in den Jahren 1972-2003 eine Gesamtreform des Strafrechtes verwirklicht, bei der das Wirtschaftsstrafrecht einen zentralen Teil gebildet hat. Von der Gesamtreform steht die Überprüfung einiger Vorschriften des Besonderen Teils des Strafgesetzes sowie die gesamte Kapitelsystematik des Besonderen Teils noch aus, aber ansonsten konnte die Rekodifizierung des aus dem Jahre 1889 stammenden Strafgesetzes im Jahre 2003 zu einem faktischen Abschluss gebracht werden.' Die Wirtschaftsstrafvorschriften wurden in den Jahren 1990, 1995 und 2003 in drei Gesetzgebungspaketen reformiert. Die grundlegenden Vorschriften über die strafrechtliche Verantwortung in der Form der echten Kriminalstrafbarkeit juristischer Personen finden sich im 9. Kapitel vom reformierten fStGB (GBl. 743/1995). Die wichtigsten den Allgemeinen Teil des Strafrechts betreffenden Vorschriften wurden in 2003 novelliert.

Die Ziele, Wirkungen und Grenzen des Wirtschaftsstrafrechts haben auch die Regelung der strafrechtlichen Verantwortung juristischer Personen beeinflusst. Die Ziele des reformierten Wirtschaftsstrafrechtes sind vor dem Hintergrund der allgemeinen Ziele der Gesamtreform des Strafrechtes zu sehen. Die wichtigste Aufgabe, die der Reformarbeit des Strafrechtskomitees gestellt worden war, hat darin bestanden, Überlegungen darüber anzustellen, was strafbar sein sollte und wie streng die einzelnen Delikte zu bestrafen sind. Für die Bestimmung der Strafbarkeit der Taten und der festzusetzenden Strafdrohungen wurde ein Modell vorgebracht, nach dem zuerst die Nachteiligkeit und Vorwerfbarkeit der Tattypen zu beurteilen und dann die Vor- und Nachteile einer eventuellen Kriminalisierung im Vergleich zu den übrigen Regulierungsalternativen abzuwägen sind. Das Komitee betonte die das strafrechtliche System kennzeichnende Wirkung: die mittelbare Wirkung und die symbolische Bedeutung der Strafdrohungen. Mit den Strafvorschriften wird aufgezeigt, welche die für die Gesellschaft zentralen Verbote und Gebote seien. Durch die Existenz der Strafdrohungen und ihre Anwendung in der Praxis wird die autoritative Missbilligung der Gesellschaft ausgedrückt und somit Einfluss auf die Herausbildung der Rechts- und Moralvorstellungen der Bürger genommen. ${ }^{*}$

Es gab kein besonderes Land, dessen Regelung als Vorbild für die Einführung der echten Kriminalstrafbarkeit juristischer Personen in Finnland gewesen wäre. Dagegen wurde ein punitives administratives Bußgeld - die Buße für Wettbewerbsverstöße - gemäß dem Vorbild des Wettbewerbsrechts der Europäischen Gemeinschaft in einem Gesetz über die Wettbewerbseinschränkungen (GBl. 480/1992) in Gebrauch genommen. Jedoch war es ein großer Mangel in Finnland, dass diejenigen punitiven administrativen Sanktionen, die besonders im Wettbewerbs- und Wertpapiermarktrecht wegen der Vorbildwirkung der EU in Gebrauch genommen worden sind, bei der Gesamtreform des Strafrechts keine reale Beachtung fanden und darum die Vor- und Nachtteile der punitiven administrativen Sanktionen im Verhältnis zu Kriminalstrafen im Gebiete des Wirtschafstrafrechts nicht systematisch erörtert wurden. ${ }^{{ }^{5}}$

Die estnische Regelung in der Strafrechtsreform 2002 - die Kriminalstrafbarkeit juristischer Personen - beruht auf der Lösung des französischen Code pénal. Schon in der Gesetzesänderung im 1992 wurde nach dem Muster des deutschen Strafrechts die entsprechende Verantwortlichkeit in den Rahmen des Ordnungswidrigkeitsrechts eingeführt. ${ }^{* 6}$

Strafgesetz (eStGB): https://wipolex.wipo.int/en/text/432563 (consolidated text of Jan. 10/2017). Die deutsche Übersetzung vom fStGB ist auch publiziert: Das finnische Strafgesetz. Deutsche Übersetzung und Einführung von K. Cornils, D. Frände und J. Matikkala. Max-Planck-Institut für ausländisches und internationales Strafrecht. Berlin: Duncker \& Humblot 2006.

4 Siehe Komiteebericht 1976:72. Helsinki 1977, Kapitel II-IV. Siehe auch im allgemeinen R. Lahti / K. Nuotio (Hrsg.), Criminal Law Theory in Transition / Strafrechtstheorie im Umbruch. Helsinki: Finnish Lawyers` Publishing Company 1992; R. Lahti, Das Wirtschaftsstrafrecht in der Gesamtreform des Strafrechts. - Wirtschaftsstrafrecht - Dogmatik, Rechtsvergleich, Rechtstatsachen. Festschrift für Klaus Tiedemann. Köln: Carl Heymanns Verlag 2008, S. 61-77 (auch im Aufsatzsammlung R. Lahti, Zur Kriminal- und Strafrechtspolitik des 21. Jahrhunderts. Berlin: de Gruyter 2019, S. 201-221. - DOI: https:// doi.org/10.1515/9783110647990).

5 Siehe näher R. Lahti, Das moderne Strafrecht und das ultima-ratio-Prinzip. - Festschrift für Winfried Hassemer. Heidelberg: C. F. Müller Verlag 2010, S. 439-448 (auch im Aufsatzsammlung R. Lahti, Zur Kriminal- und Strafrechtspolitik des 21. Jahrhunderts. Berlin: de Gruyter 2019, S. 107-118. - DOI: https://doi.org/10.1515/9783110647990).

6 Siehe eStGB § 14; Pikamäe, Sootak (Fn. 1), S. 154-155. Zur internationalen Entwicklung vgl. insbesondere M. Engelhart, Corporate Criminal Liability from a Comparative Perspective. - D. Brodowski et al. (eds.), Regulating Corporate Criminal Liability. Switzerland: Springer International Publishing 2014, S. 53-76. - DOI: https://doi.org/10.1007/978-3-319-059938_6. 


\section{Bereich der Verantwortlichkeit: Spezialitäts- oder Universalitätsprinzip?}

Über die in Frage kommenden, bei der Tätigkeit der juristischen Person begangenen Delikte wurden separate Vorschriften erlassen (fStGB 9:1.1). Zu solchen Delikten wurden in der Anfangsphase die Bestechungsdelikte (fStGB 16:18; 563/1998), die Beihilfedelikte (fStGB 29:10), die meisten von den Gewerbedelikten im Kapitel 30 vom StGB (fStGB 30:13), die Geldwäsche und die sonstigen schweren Hehlereidelikte (fStGB 33:8), die Rationierungsdelikte und der Schmuggel (fStGB 46:14) sowie die Umweltdelikte (fStGB 48:9) bestimmt. Man erachtete den Bedarf dieser neuen Verantwortungsform für am offenbarsten bei der im weiten Sinne definierten Wirtschaftskriminalität, aber nicht alle Deliktstypen dieser Kriminalität - zum Beispiel Steuer-, Buchhaltungs-, Schuldner- oder Arbeitsdelikte - wurden in den Kreis der neuen strafrechtlichen Verantwortlichkeit mit einbezogen. In den Begründungen zur Gesetzesänderung wurde die Bedeutung der Erwägung der kriminalpolitischen Notwendigkeit und des Behutsamkeitsprinzips betont.

Seitdem ist der Bereich der strafrechtlichen Verantwortlichkeit der juristischen Person insbesondere aufgrund der Vorschriften der Europäischen Union wesentlich erweitert; der Anwendungsbereich enthält nun nach der Geltungsdauer über 20 Jahre mehr als 8o Straftattypen, und diese Verantwortungsform ist auch auf Arbeitsschutzdelikte (fStGB 47:9) ausgedehnt worden. Die in den Gesetzesbegründungen geäuBerte Annahme, dass man nur selten Körperschaftsgeldstrafen verhängen würde, traf besonders in den Anfangsjahren zu. Damals wurden vor den Gerichten nur einige wenige Fälle der kollektiven Verantwortung gemäß dem Kapitel 9 vom fStGB verhandelt. Zum Teil hatte darauf der Umstand einen Einfluss, dass der Staatsanwalt bis zur Gesetzesänderung des Jahres 2003 reichlich Ermessensspielraum hinsichtlich einer sanktionsartigen Verfolgung hatte. Danach ist es häufiger geworden, dass juristische Personen strafrechtlich verantwortlich gemacht werden, und u. a. die Präjudizien OGH (KKO) 2008:33 und 2009:1 des Obersten Gerichtshofs haben diesbezüglich eine steuernde Wirkung ausgeübt. Bis Ende des Jahres 2017 hatte man 411 Male zur Körperschaftsgeldstrafe verurteilt, und in meisten Fällen für Arbeitsschutzdelikte."7

Auch in Estland ist das Spezialitätsprinzip gültig, weil laut des §14 Abs. 1 eStGB die juristische Person nur in den im Gesetz direkt vorgeschriebenen Fällen bestraft wird. Die finnische Regelung ist seit dem Beginn dem Universalitätsprinzip näher gekommen, wenn die Anzahl der Straftattypen auf welche die Kriminalstrafbarkeit der juristischen Person sich anpassen lässt, wesentlich erweitert worden ist. ${ }^{*}$

\section{Anknüpfungstat und Zurechnungsstruktur der Verantwortlichkeit}

Die Zurechnungsstruktur der echten Kriminalstrafbarkeit ist im Kapitel 9 vom fStGB nicht ganz klar. In erster Linie denkt man, dass die juristische Person aufgrund der Tat eines individuellen (in Ausnahmefällen auch anonymen) Täters bestraft wird, aber andererseits ist in der Regelung eine gewisse kollektive, „gemeinschaftliche“ Schuld oder Zurechenbarkeit erkennbar.

Als eine Grundvoraussetzung für die gemeinschaftliche Verantwortung gilt, dass eine Straftat im Rahmen der Tätigkeit der juristischen Person begangen wurde (fStGB 9:1.1). Das Delikt gilt als im Rahmen der Tätigkeit der juristischen Person begangen, wenn der Täter im Namen der juristischen Person oder zu ihren Vorteil gehandelt hat und er zur Leitung der juristischen Person gehört oder in einem Dienst- oder Arbeitsverhältnis zu ihr steht oder im Auftrag eines Vertreters der juristischen Person gehandelt hat (fStGB 9:3.1). Es ist jedoch nicht unbedingt notwendig, dass ein solcher individueller Täter ermittelt oder bestraft wird (fStGB 9:2.2).

Dazu setzt die gemeinschaftliche Verantwortung die oben genannte gemeinschaftliche Schuld (durch Identifizierung oder Geschäftsherrnhaftung) voraus. Eine zu einem gesetzmäßigen Organ oder zur sonstigen Leitung der juristischen Person gehörende Person muss an der Straftat beteiligt sein oder die Tat zugelassen haben, es sei denn, dass im Rahmen der Tätigkeit der juristischen Person nicht die gegebene Sorgfalt und Vorsicht zur Verhütung der Straftat gewahrt wurde (fStGB 9:2.1). Bei einem Ölraffineriefall

Siehe näher Alvesalo-Kuusi, Lähteenmäki (Fn. 2).

Vgl. Pikamäe, Sootak (Fn. 1), S. 155. 
(OGH 2008:33) war die Streitfrage, ob die Angeklagten einer solchen Leitung angehörten, d.h. ob sie eine ausreichende selbständige und bedeutende Beschlussfassung in der Aktiengesellschaft ausübten oder nicht.

Auch im estnischen Strafrecht ist die Anknüpfungstat der natürlichen Person als die primäre Voraussetzung der gemeinschaftlichen Verantwortung. Diese Anknüpfungstat muss - wie im finnischen Strafrecht - im Interesse der Korporation begangen sein, aber dieser Begriff sollte ausweitend ausgelegt werden ${ }^{*}$. Dazu fordert man, dass die natürliche Person ein Leitungsfunktionär oder - nach der Gesetzesänderung vom 2008 - zuständiger Vertreter ist ${ }^{* 10}$. Pikamäe und Sootak haben jene Gesetzesänderung kritisiert, weil der Begriff zuständiger Vertreter eine Abweichung von dem Prinzip bedeutet, wonach „der Anknüpfungstäter eine von den Personen sein muss, die den Willen der juristischen Person äußern und ihre Tätigkeit gestalten “*11.

Die Regelungen im finnischen und estnischen Strafrecht sind darin ähnlich, dass eine Doppelbestrafung möglich ist und dass die individuelle Organ- und Vertreterhaftung in der Praxis den Vorrang hat. Gleichzeitig mit der Einführung der strafrechtlichen Verantwortlichkeit der juristischen Person wurden bei der Reformierung des Strafgesetzes in die neuen Kapitel über Arbeits- und Umweltdelikte (fStGB 47:7 und 48:7) Regelungen über die Zuweisung der individuellen strafrechtlichen Verantwortlichkeit eingeführt. Dazu wurde im Jahre 2003 im Zusammenhang des Allgemeinen Teils des Strafgesetzes (fStGB 5:8) eine Bestimmung über „Handeln für eine juristische Person“ nach dem Modell des deutschen Strafgesetzes (§ 14) aufgenommen. ${ }^{*}$

Die Regelungen im finnischen und estnischen Strafrecht sind auch darin ähnlich, dass die Kriminalstrafbarkeit der juristischen Person nicht auf die Ausübung öffentlicher Gewalt angewandt wird (fStGB 9:1.2; eStGB § 14.3).

\section{Schuldprinzip und sein Verhältnis zur Zurechnungsstruktur}

Wie oben (4) gesagt, die Zurechnungsstruktur der Kriminalstrafbarkeit der juristischen Person ist im finnischen Strafrecht nicht ganz klar, und dasselbe gilt auch im Verhältnis zum Schuldprinzip. Die finnische Regelung bedeutet, dass die juristische Person nicht selbst als Straftäter angesehen wird ${ }^{*}{ }^{*}$. Jedoch ist das Schuldprinzip nicht nur höchstpersönlich, sondern die Regelung spiegelt Züge der gemeinschaftlichen Schuld wider: eine individuelle Straftat muss nicht nur im Rahmen der Tätigkeit der juristischen Person begangen worden sein, sondern dazu hat man innerhalb der Organisation der juristischen Person nicht genügend getan, um die Begehung der fraglichen Straftat zu verhindern. ${ }^{*} 4$

Kapitel 9 vom fStGB ist lex specialis, wenn es die Voraussetzungen der Kriminalstrafbarkeit in dessen Paragraphen 2 und 3 bestimmt. Die estnische Regelung ist verschieden. Das Schuldprinzip gehört in Estland zu den grundgesetzlichen Prinzipien, und die Deliktsstruktur vom eStGB macht keinen Unterschied zwischen natürlichen und juristischen Personen. Die Schuldfähigkeit ausschließenden Umstände im Kapitel 2 Abschnitt 3 eStGB gelten folglich formell sowohl für die natürliche als auch für die juristische Person. ${ }^{*} 5$ Nach $\S 37$ eStGB e contrario ist eine juristische Person schuldfähig, wenn sie rechtsfähig ist.

In der estnischen Strafrechtsdoktrin hat man den doppelgeschichteten Charakter des Tatbestandes sowie des Schuldprinzips erörtert. Das Prinzip der derivativen Verantwortung (die Anknüpfungstat der

9 Sootak, Elkind (Fn. 1), S. 431-433.

10 Siehe eStGB $\S 14.1:$ ”...by its [legal person's] body, a member thereof or by a senior official or competent representative”; Sootak, Elkind (Fn. 1), S. 425-430.

11 Pikamäe, Sootak (Fn. 1), S. 157.

12 Näher über das Verhältnis zwischen der strafrechtlichen Verantwortung der juristischen Person und der Organ- und Vertreterhaftung in Finnland, siehe Lahti, Festschrift für Keiichi Yamanaka (Fn. 2); R. Lahti, Finland National Report. - K. Ligeti, A. Marletta (eds.), Punitive Liability of Heads of Business in the EU: A Comparative Study. Italia: Wolters Kluwer 2019, S 5-36.

13 Tolvanen 2009 (Fn. 2) drückt das wesentliche Zurechnungsprinzip in folgender Weise aus: „the acts of the individual offender are under certain conditions attributed to the legal person, not as acts of the legal person but as acts of the individual for the company“. Siehe auch im allgemeinen Engelhart 2014 (Fn. 6), S. 58.

14 So die Formulierung von Frände 2001 (Fn. 2), S. 229.

15 Pikamäe, Sootak (Fn. 1), S. 158. 
natürlichen Person) ist nicht genug: Einerseits sind auf der Ebene der objektiven Tatbestandsmäßigkeit die Voraussetzungen des Interesses der juristischen Person sowie die besondere Stellung des Täters (am meisten die Stellung eines Leitungsfunktionärs). Andererseits fordert man auf der Ebene des Schuldprinzips die Vermeidbarkeit der Anknüpfungstat (aktives Tun oder Unterlassen), aber in Einzelheiten ist der Inhalt dieser Forderung mehrdeutig. ${ }^{* 16}$

Ein unterschiedliches Detail in der estnischen Doktrin im Verhältnis zur finnischen Doktrin betrifft die theoretische Konstruktion der Verantwortung, wenn die Anknüpfungstat von einem Durchschnittsarbeiter gemacht worden ist. Im estnischen Strafrecht ist die Verantwortung der juristischen Person mit der Rechtsfigur der mittelbaren Täterschaft - durch eine Tatherrschaft mittels organisatorischer Machtapparate - begründbar, weil im finnischen Strafrecht eine extensive (Mit)Täterschaft befürwortet wirdt. Nach der neuen, engen gesetzlichen Formulierung von der mittelbaren Täterschaft (fStGB 5:4) gibt es einen besonderen Bedarf für diese weite Auslegung der Täterschaft im Verhältnis zu Straftaten, die im Rahmen der Tätigkeit von juristischen Personen begangen werden (siehe dazu fStGB 3:3.2, 5:8 und 9:2.1). ${ }^{* 17}$

\section{Sanktionen}

Kapitel 9 vom fStGB enthält Bestimmungen mit folgenden Titeln: Körperschaftsgeldstrafe (§ 5), Grundlagen für die Bemessung der Körperschaftsgeldstrafe (§ 6), Absehen von einem Strafantrag (§ 7), Gemeinsame Körperschaftsgeldstrafe (§ 8) und Vollstreckung der Körperschaftsgeldstrafe (§ 10). Die Körperschaftsgeldstrafe ist die einzige Strafe, die für die juristische Person im Gebrauch ist. Die Einziehung lässt sich auch auf die juristische Person anwenden, aber diejenige gehört zu den strafrechtlichen Sicherungswahrnahmen und nicht zu den Strafen.

Der Mindestbetrag der Körperschaftsgeldstrafe beträgt 850 Euro und ihr Höchstbetrag 850 ooo Euro. Diese Beträge sind unverändert seit dem Erlassen des Kapitels (1995) gewesen. Darum besteht jetzt eine Rechtslage, die vom Gesichtspunkt des Verhältnismäßigkeitsprinzips der Kritik ausgesetzt ist, weil die strengsten punitiven administrativen Bußgelder u.a. für Wettbewerbsverstöße viel höher sein können.

Im estnischen Strafrecht betragen nach der Gesetzesänderung in 2014 die Mindest- und Höchstbeträge der Körperschaftsgeldstrafe 4 ooo Euro und 16 ooo ooo Euro (eStGB § 44.8). Es besteht also ein großer Unterschied zwischen Finnland und Estland. Man bemesst die Geldstrafe des gehörigen Unternehmens im Verhältnis zu seinem Umsatz (eStGB § 44.9), was auch sich von der finnischen Regelung unterscheidet. ${ }^{* 18}$ Gewisse Nebenstrafen sind auch für die juristische Person im Gebrauch (eStGB § 55 ${ }^{1}$ ).

\section{Zusammenfassung}

Die gesetztechnischen Lösungen zur Einführung der Kriminalstrafbarkeit der juristischen Person sind in Finnland (1995) und Estland (2001) verschieden gewesen. Ähnlichkeiten zeigen sich u.a. darin, dass in beiden Ländern diese Regelung im Zusammenhang der Gesamtreform des Strafgesetzes verwirklicht wurde. Auch in prinzipiellen Hinsichten - betreffend die Zurechnungsstruktur und das Schuldprinzip - sind die Regelungen de facto einander nah. Unterschiede bemerkt man u.a. darin, dass in Finnland die Regelung der individuellen Organ- und Vertreterhaftung in der Gesamtreform revidiert wurde und dass die Beteiligungsdoktrin zwischen diesen Ländern sich voneinander unterscheidet. Andererseits ist das Strafmaß der Körperschaftsgeldstrafe in Estland in zeitgemäßer Weise erhöht worden. Für diese beiden Länder der Europäischen Union ist es eine Zukunftsfrage, ob man eher punitive administrative Sanktionen statt der Körperschaftsstrafen einführen und verwirklichen sollte ${ }^{* 19}$.

16 Siehe näher Pikamäe, Sootak (Fn. 1), S. 156-160; Sootak, Elkind (Fn. 1), S. 425-431. Nach der Gesetzesergänzung in 2014 (eStGB § 371) ist ein solcher Grund für den Ausschluss der Schuld der juristischen Person expressis verbis in Kraft.

17 Vgl. einerseits Pikamäe, Sootak (Fn. 1), S. 156, 160 mit Hinweis auf die Dissertation von P. Randma; und andererseits R. Lahti, Festschrift für Yamanaka (Fn. 2), S. 143-146.

18 Vgl. mit der ursprünglichen Regelung in Sootak, Elkind (Fn. 1), S. 433.

19 In Finnland hat eine Arbeitsgruppe des Ministeriums für Justiz in einem neuen Bericht geklärt, wie die Regelung der punitiven administrativen Sanktionen entwickelt werden sollte. Siehe Reihe der Berichte des Ministeriums für Justiz, 52/2018. 